\title{
Glioblastomas: clasificación, caracterización molecular y vías de señalización comúnmente alteradas
}

\section{Glioblastomas: classification, molecular characterization and commonly altered signaling pathways}

Pág. 106, 115

Recibido: 09-06-2020

Aceptado: 25-06-2020

Dra. María Catalina Camacho Alvarado ${ }^{1}$

Dra. Daniela Taboada Mora²

1,2. Médico Cirujano General, trabajador independiente. San José,Costa Rica.

\section{RESUMEN}

EI Glioblastoma Multiforme(GBM)es la neoplasia primaria maligna del Sistema Nervioso Central (SNC) más frecuente a nivel mundial. Este tiene una de las tasas de supervivencia más bajas de cualquier tumor cerebral a pesar de avances en técnicas diagnósticas, mayor entendimiento de su génesis tumoral y la existencia de terapias dirigidas. Se modifica la Clasificación de Tumores del SNC de la Organización Mundial de la Salud (OMS) de 2016 debido a la relevancia de incluir parámetros moleculares además de la caracterización histológica clásica para su diagnóstico, las principales vías de señalización alteradas y el biomarcador Metil-guanina metiltransferasa (MGMT).

\section{ABSTRACT:}

Glioblastoma Multiforme (GBM) is the most frequent primary malignant neoplasm of the Central Nervous System (CNS) worldwide. It has one of the lowest survival rates of any brain tumor despite advances in diagnostic techniques, greater understanding of its tumor genesis and the existence of targeted therapies. The 2016 World Health Organization (WHO) CNS Tumor Classification is modified due to the relevance of including molecular parameters in addition to the classic histological characterization for diagnosis, the main altered signaling pathways and the methyl-guanine methyl transferase (MGMT) biomarker. 


\section{PALABRAS CLAVE}

Glioblastoma, neoplasias del sistema nervioso central, clasificación, transducción de señal, biomarcadores.

\section{KEYWORDS}

Glioblastoma, central nervous system neoplasms, classification, signal transduction, biomarkers.

\section{INTRODUCCIÓN}

El Glioblastoma Multiforme (GBM) es el tumor primario maligno del Sistema Nervioso Central (SNC) más frecuente a nivel mundial. Corresponde al $14 \%$ de todos los tumores de SNC y al $48 \%$ de todos los tumores malignos de SNC. Estos últimos con una incidencia estimada en 7.08 por cada 100.000 habitantes según estadísticas del Registro Central de Tumores Cerebrales de los Estados Unidos (CBTRUS, por sus siglas en inglés)(1)(Tabla 1).

La heterogeneidad de sus componentes, así como su alta resistencia a tratamiento lo colocan entre las neoplasias más agresivas y de peor pronóstico(2). La mediana de supervivencia posterior al diagnóstico es de 12 meses y la tasa de supervivencia a 5 años es de $6.8 \%(1)$, contribuyendo de manera desproporcional a la mortalidad y morbilidad por cáncer(3).

\begin{tabular}{|lcc|}
\hline Incidencia de Tumores de Sistema Nervioso Central" \\
\hline & $\begin{array}{c}\text { Por cada } \\
100.000 \\
\text { habitantes }\end{array}$ & $\%$ \\
\hline Global & 3.41 & 100 \\
\hline $\begin{array}{l}\text { Tumores } \\
\text { malignos }\end{array}$ & 7.08 & 30.02 \\
\hline $\begin{array}{l}\text { Tumores } \\
\text { Benignos }\end{array}$ & 16.33 & 69.8 \\
\hline
\end{tabular}

El GBM tiene una edad promedio de aparición de 64 años, siendo poco común en niños. La incidencia es 1.6 veces mayor en hombres que en mujeres y 2.0 veces mayor en caucásicos en comparación con africanos y afroamericanos, con menor incidencia en asiáticos e indios americanos(4).

Costa Rica presentó una de incidencia de 1,3 por cada 100,000 habitantes para 2015, según datos oficiales del Ministerio de Salud. En promedio, entre el 2011 y el 2015, se reportaron 51 casos por año entre todos los grupos etarios.

La mayor incidencia se presenta en la población adulta, comportamiento similar al establecido en estadísticas internacionales. Sin embargo, no se cuenta con datos nacionales oficiales de la sub-clasificación de los GBM, de acuerdo con los criterios moleculares establecidos por la Organización Mundial de la Salud (OMS)(5).

EI GBM se describe clásicamente como un tumor de origen glial, de características infiltrativas, con nucleación atípica, alta actividad mitótica, proliferación vascular extensa y presencia de necrosis(6). Por lo general, tiene una localización supratentorial, con alguna incidencia a nivel cerebelar. Su presentación clínica depende de la localización anatómica, así como de sus características histológicas y moleculares.(7).
Tabla 1. Incidencia de tumores malignos y benignos de Sistema Nervioso Central según datos del CBTRUS(1). * Ajustada por edad. 


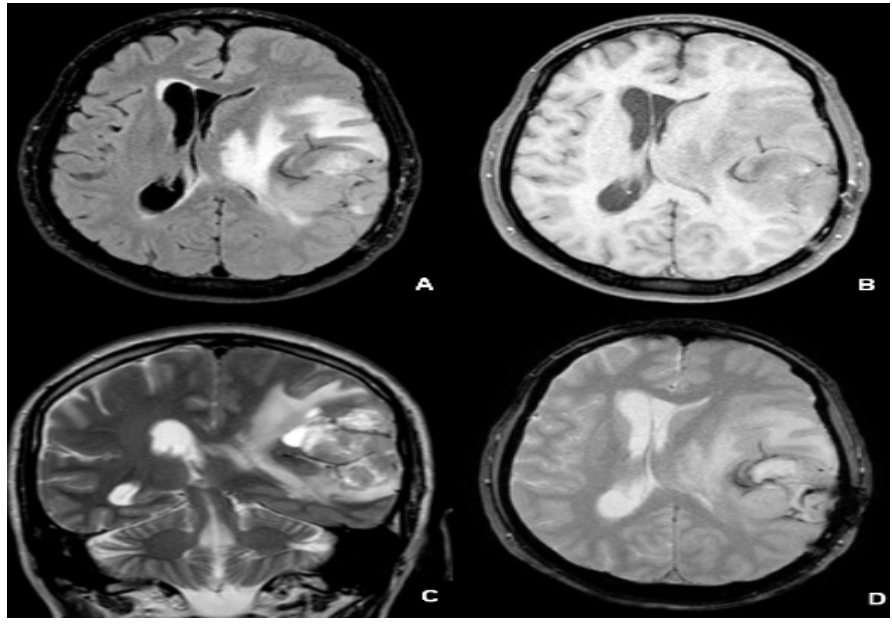

Imagen 1. Imagen de Resonancia Magnética de GBM, lesión tumoral parietal izquierda. A y B. Cortes axiales en Flair y $\mathrm{T} 1$ sin contraste, respectivamente. Lesión intraparenquimatosa heterogénea con efecto de masa, edema perilesional (flechas amarillas en A), desviación de línea media y compresión de ventrículos laterales (flecha negra en imagen B). C. Corte Coronal en T2. Herniación subfalcial leve (flecha blanca). D. Corte axial en T2. Edema perilesional (flecha amarilla). Cortesía del Centro Nacional de Resonancia Magnética (CNRM).

Según la Clasificación de Tumores de SNC de la OMS realizada en 2007, el GBM se definió como una entidad de origen astrocítico, Grado IV, con dos variantes: el GBM de células gigantes y el Gliosarcoma. Desde entonces, se vinculan algunas alteraciones genéticas comunes en la génesis tumoral del GBM, tales como amplificación del receptor del factor de crecimiento epidérmico (EGFR por sus siglas en inglés, también conocido como HER1), supresión homocigota del gen p16INK4a, y mutaciones gen PTEN y pérdida de la heterocigosidad en el cromosoma $10 q(8)$.

Si bien se reconocían estas características moleculares en los GBM, no constituyeron criterios diagnósticos, sino que fueron utilizadas como marcadores de proliferación celular y determinantes de comportamiento biológico(9). En la última clasificación de Tumores de SNC de la OMS publicada en 2016, se integran por primera vez criterios moleculares, cambio de particular relevancia para la definición del GBM(2).

A pesar de la integración histo-molecular para el diagnóstico del GBM y comprenderse de una manera más amplia las vías de señalización comúnmente alteradas, existen pocos cambios significativos en el abodaje médico-quirúrgico en las últimas dos décadas. El estándar de tratamiento implica resección tumoral máxima seguida de radioterapia, además de quimioterapia con Temozolomida(TMZ)(10), régimen establecido en el ensayo clínico de 2004 EORTC-NCIC. Si bien este protocolo es el de mayor sustento científico hasta el momento, la heterogeneidad molecular y mecanismos de resistencia celular del GBM requieren de fármacos que superen su complejidad(3).

\section{DESARROLLO}

La Clasificación de Tumores del Sistema Nervioso Central de la Organización Mundial de la Salud, edición 2016, incluye por primera vez parámetros moleculares además de la caracterización histológica clásica. Previamente, las similitudes tumorales establecidas dependían únicamente del análisis microscópico bajo tinción de hematoxilina/eosina, expresión inmunohistoquímica y rasgos ultraestructurales. Esta clasificación se planteó a partir de las guías de incorporación de hallazgos moleculares en el diagnóstico de tumores cerebrales, establecidas por la Sociedad de Neuropatología en 2014(2). 

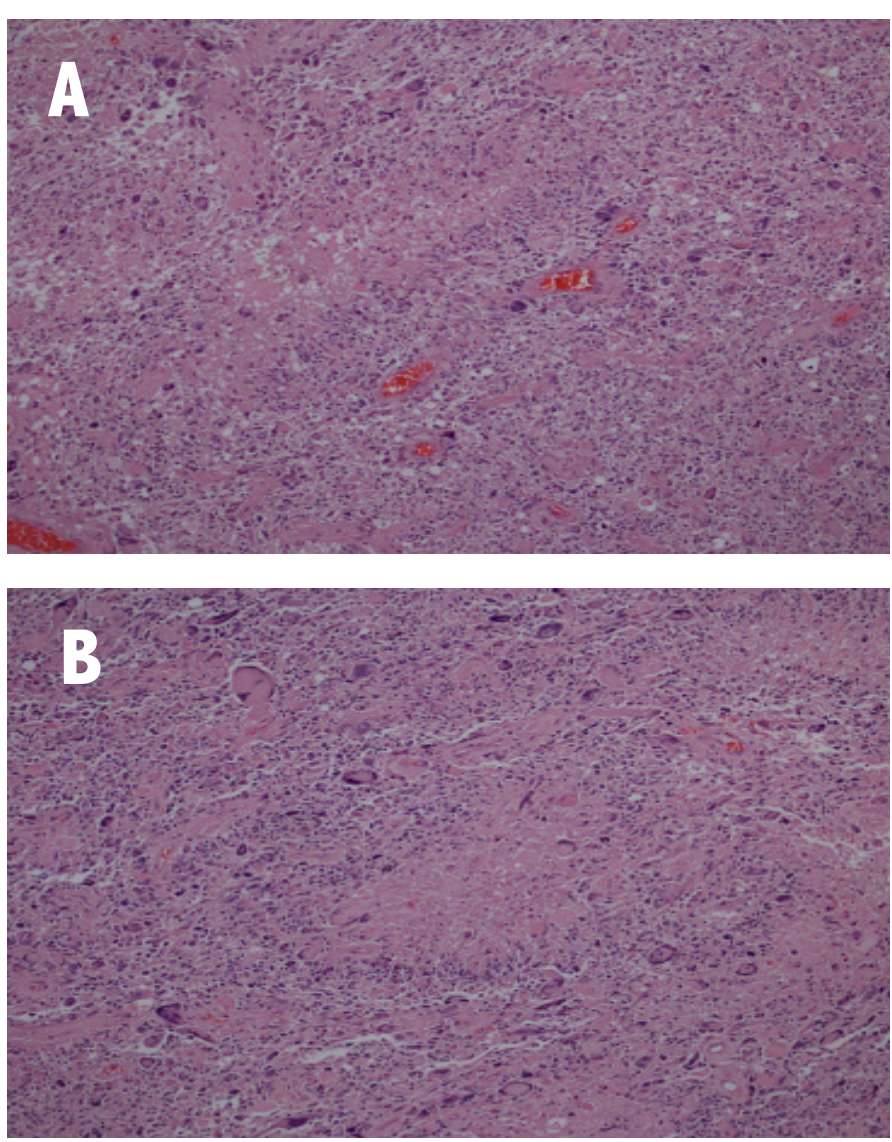

Imagen 2. Cortes histológicos de GBM. A y B. Se observa aumento de la celularidad y presencia de importante pleomorfismo celular, necrosis en empalizada y proliferación microvascular. Cortesía Servicio de Anatomía Patológica, Hospital México.

En la actualización realizada se definen entidades nuevas, subclasificación o variantes y algunos patrones histológicos reconocibles sin mayor significancia clínico-patológica. Se incorporan así el GBM isocitrato-deshidrogenasa (IDH) silvestre o "wild-type" y el GBM isocitrato-deshidrogenasa mutado, en ausencia de codeleción en regiones $1 \mathrm{p} / 19 \mathrm{q}$. Se asigna adicionalmente una categoría NOS, "no otherwise specified" por sus siglas en inglés, para los tumores no tipificables y aquellos casos donde no se cuente con pruebas moleculares(2).

Aproximadamente el $90 \%$ de los glioblastomas son IDH silvestres, tumores primarios y observados con mayor frecuencia en pacientes mayores de 55 años. Cerca del 10\% de los casos presentan mutación en la IDH, típicamente secundarios a gliomas IDH-mutados de menor grado y característicos de población adulta joven(6). La mutación en la IDH es una de las primeras alteraciones genéticas detectables y principales precursoras de la génesis tumoral de los gliomas(11).

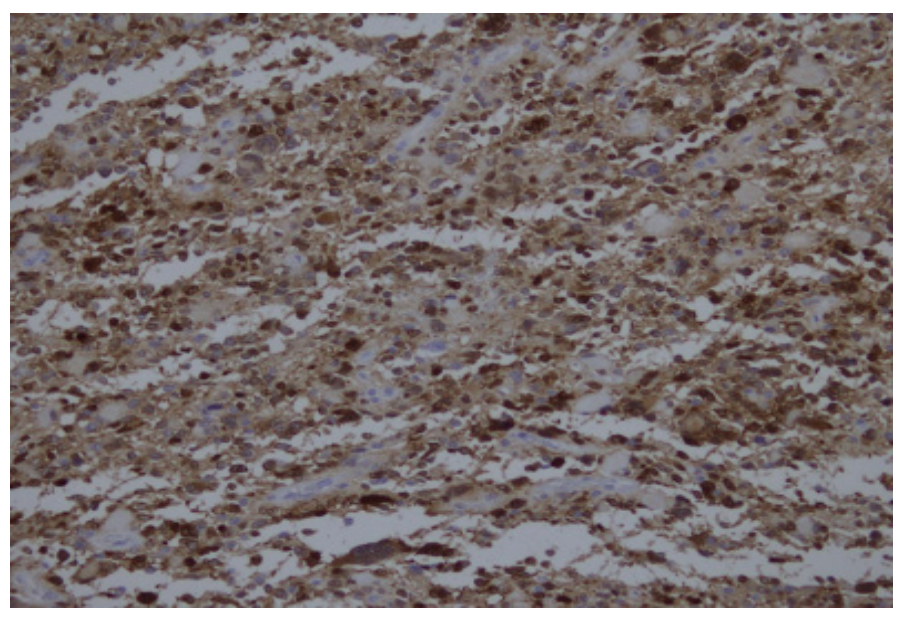

Imagen 3. GBM IDH-1 positivo, inmunohistoquímica. Cortesía Servicio de Anatomía Patológica, Hospital México.

Existen distintos perfiles genéticos que culminan en la expresión anómala de la enzima IDH. Por lo general los astrocitomas difusos o anaplásicos IDH-mutados y los GBM IDH-mutados secundarios a ellos, tienen vías de génesis tumoral comunes. Asimismo, presentan un comportamiento biológico similar y mutaciones en los genes ATRX y TP53. Por otro lado, el GBM IDH-salvaje presenta alteraciones en tres vías principales, descritas más adelante.

En términos fisiológicos, la IDH es conocida por su rol en el ciclo de Krebs como catalizadora de la descarboxilación oxidativa del isocitrato, resultando en alfa-cetoglutarato y dióxido de 
carbono. Las isoformas IDH1 e IDH2 codifican proteínas citosólicas y mitocontriales, respectivamente(6). Ambas isoformas tienen un papel primordial en el metabolismo celular, regulación epigenética, estados Redox y de reparación del ácido desoxirribonucleico (ADN)(12).

Mutaciones puntuales en IDH1 e IDH2 ocasionan sustitución de arginina a histidina o a lisina, respectivamente. Dicha sustitución altera la actividad catalítica de manera que producen niveles altos del oncometabolito 2-hidroxiglutarato (2-HG) en lugar de alfa-cetoglutarato(13). EI 2-HG funciona como un inhibidor competitivo de la alfa-cetoglutarato, deteriorando la función de dioxigenasas-alfa-cetoglutarato dependientes como las histonas y las ADN-desmetilasas (enzimas TET). Esto conduce a alteraciones de metilación del ADN a lo largo del genoma(14).

Las mutaciones en la IDH1 e IDH2 en los casos de GBM del adulto predicen una supervivencia mayor en comparación con los GBM tipo silvestre(10). A su vez, la alteración enzimática de ambas isoformas se asocia a un periodo libre de enfermedad más prolongado y una mejor respuesta a quimioterapia con $\mathrm{TMZ}(6)$.

También se agrega en 2016 el GBM tipo epitelioide, que junto con el GBM de células gigantes y el gliosarcoma, forman parte de la subclasificación del subtipo silvestre. La variante epitelioide presenta células grandes con citoplasma eosinofílico y nucléolos prominentes, que se asemejan a las células del melanoma, y células rabdoides. Es más frecuente en niños y adultos jóvenes portando una mutación en el gen BRAF V600E. Usualmente carecen de otras características típicas del GBM tipo silvestre, como mutaciones en el receptor del factor de crecimiento epidérmico (EGFR por sus siglas en inglés) y pérdidas en el cromosoma 10(6).

La variante GBM de células pequeñas se caracteriza por células gliales monofórmicas(15), mientras que el Gliosarcoma presenta componentes gliales y mesenquimales. Radiológica y clínicamente estas variables son indistinguibles y se requiere diagnóstico histopatológico. Los patrones corresponden a: GBM de células pequeñas, GBM de células granulares y de componente neural primitivo.

Desde el punto de vista clínico, se hace referencia al origen primario y secundario, sin embargo, quedan en desuso como criterios diagnósticos. El uso de marcadores moleculares genera un reordenamiento de las familias tumorales y se conoce de previo que algunas entidades serán descartadas conforme se avance en técnicas biomoleculares. Esta actualización no incluye una técnica específica para el diagnóstico molecular y lo deja a criterio médico y a disponibilidad del centro involucrado.

\section{VÍAS DE SEÑALIZACIÓN ALTERADAS EN GBM}

Datos de The Cancer Genome Atlas (TCGA) muestran alteraciones en 3 vías centrales de señalización específicas:

\section{VÍA P14ARF-MDM2-MDM4-P53}

La proteína p53, codificada en el gen TP53 en el cromosoma 17p13.1 tiene un rol fundamental en la supresión tumoral. En respuesta a señales de estrés como daño del ADN, el p53 induce reparación de ADN, arresto del ciclo celular y apoptosis celular. Un $86 \%$ de los GBM tiene desregulación de la vía p53 por mutación o deleción del gen TP53 (28\%), amplificación

\section{(c) $)(1) \Theta$}


MDM2/4 (15\%) reguladores negativos de p53 y/o deleción o mutación de CDKN2A (58\% expresa p14ARF, regulador negativo de MDM2/4) (16). Las alteraciones de TP53 son mutualmente excluyentes con cambios MDM2/4 y son más comunes en glioblastomas secundarios que en primarios (68\% vs $28 \%$ ). La pérdida de p14ARF no muestra predilección por ningún subtipo de $\operatorname{GBM}(20)$.

No se conoce bien la implicación pronóstica de la mutación de p53 en GBM, principalmente por la diversidad de mutaciones que estos presentan. En gliomas IHD mutados se encuentra frecuentemente la mutación de p53 y se asocia a un mejor pronóstico. Por el contrario, los glioblastomas primarios no se suele encontrar la mutación y conllevan un peor pronóstico(6).

\section{VÍA RTK-RAS-PI3K}

Varios receptores de superficie de tirosina quinasa (RTKs), como el receptor de crecimiento epidérmico (EGFR) y el receptor de factor de crecimiento derivado de plaquetas A (PDGFRA), se encuentran sobreexpresados en gliomas de alto grado y muestran distintas alteraciones en los subtipos de GBM clásico y proneural.

Elgen delEGFRse localizaenel cromosoma 7p12 y codifica un RTK que promueve la proliferación celular que tiene efecto en las vías MAPK y PI3K. Un $50 \%$ de los GBM primarios tienen sobreexpresión del EGFR por amplificación de su gen correspondiente, alteración mutuamente excluyente con codeleción $1 p / 19 q$ y mutaciones en el gen de la IDH. Los reordenamientos del gen EGFR, que comúnmente involucran la variante EGFRvIll con una sobrexpresión en el marco de los exones 2 a 7 , dan como resultado la activación de vías de señalización mitogénicas.
Las translocaciones comunes fusionan porciones de EGFR a otros asociados genéticos, siendo EGFR-SEPT14 (septin 14) la fusión génica funcional más común. Estas alteraciones son altamente sugestivas de GBM, especialmente en pacientes mayores. A pesar de ello, no hay evidencia suficiente para utilizar la amplificación de EGFR o la mutación EGFRvIll como pronóstico para pacientes con GBM.

El gen del PDGFRA se localiza en el cromosoma $4 \mathrm{q} 12$ y codifica un RTK similar al EGFR también vinculado en la proliferación del GBM. Este receptor de superficie se encuentra mutado en un $30 \%$ de los GBM, con múltiples reordenamientos reconocidos. La isoforma del receptor P PDGFRA8,9 (deleción de exones 8 Y 9) es frecuente resulta en activación constitutiva. La fusión génica entre el receptor del dominio de inserción de quinasa (KDR) y el gen PDGFRA muestra una actividad tirosina quinasa aumentada constitutivamente.

También se presenta la amplificación del PDGFRA y muestra una reducción importante en la sobrevida media, solamente en el subgrupo IDH1 mutado. Si bien se reconoce la importancia del PDGFR en la proliferación del GBM, tampoco se reconoce su significancia pronóstica.

MET es un proto-oncogen inducido por hipoxia y un receptor de superficie de tirosina quinasa para el factor de crecimiento del hepatocito. Es común su amplificación en GBM y estudios genómicos de a nivel de células individuales mostraron que una pequeña fracción de células de glioblastomas tiene amplificación focal de c-MET(21). Múltiples procesos celulares en los gliomas se encuentran regulados por el MET, tales como su proliferación, supervivencia 
y migración. La sobreexpresión de MET en los GBM se asocia a mal pronóstico y mayor inva$\operatorname{sividad}(22)$.

El homólogo de la fosfatasa y tensina (PTEN) es un gen supresor tumoral ubicado en el cromosoma 10q23, codifica una proteína que actúa como regulador negativo de la vía de señalización PI3K/AKT/objetivo mamífero de la rapamicina (mTOR). Es común encontrar mutaciones o deleciones en el PTEN en glioblastomas primarios del adulto(17).

La pérdida de heterocigosidad ( $\mathrm{LOH}$, por sus siglas en inglés) en el cromosoma 10 es la alteración cromosómica más frecuente en los glioblastomas primarios, observándose en un $80 \%$. La deleción en el locus PTEN es de las regiones del cromosoma 10 más comúnmente eliminados(23). La pérdida de la expresión de PTEN por IHC no confiere independientemente un peor pronóstico en los casos de glioblastoma recién diagnosticados tratados con temozolomida (TMZ). Por el contrario, LOH del cromosoma 10 es predictivo de una supervivencia más cor$\mathrm{ta}(24)$.

Por FISH se puede identificar fácilmente las aberraciones citogenéticas en PTEN, incluidas las deleciones homocigóticas y hemizigotas, así como la pérdida total del cromosoma 10. Alternativamente, se pueden utilizar métodos basados en PCR. La evaluación de la pérdida de PTEN por parte de IHC aún no es confiable(17). La fosfatidilinositol-4,5-bisfosfato 3-quinasa representa una familia de proteínas con una amplia variedad de funciones, que incluyen el crecimiento celular, la supervivencia y la diferenciación. Esta familia PI3K se subdivide en varias clases de proteínas. Las proteínas de clase I, como PIK3CA y PIK3R1, tienen funciones cata- líticas y reguladoras distintas, respectivamente, como parte de la vía PI3K / AKT / mTOR. Esta cascada de señalización es activada por RTK como EGFR y es inhibida por PTEN(25).

Varios miembros de esta vía, incluidos RTK, PI3K, AKT y mTOR, son los blancos princiapales para la inhibición farmacológica En los glioblastomas, la activación de la vía PI3K / AKT / mTOR está asociada con la resistencia a la quimioterapia con TMZ(26). La activación de esta vía se ha asociado con una supervivencia global significativamente menor en pacientes con glioblastoma(6).

El estudio de estas vías da luz a la complejidad de la tumorogenesis del GBM. Al mismo tiempo, permite el establecimiento de marcadores pronósticos y de resistencia a tratamiento y la elaboración de terapias dirigidas. Entre los biomarcadores más prometedores se encuetra el de la enzima metil-guanina metiltransferasa (MGMT). La metiltransferasa de ADN ha demostrado tener valor predictivo en pacientes con GBM considerados candidatos para tratamiento con $\mathrm{TMZ}(10)$.

El mecanismo de acción de la TMZ implica la metilación de la posición $\mathrm{O} 6$ de la guanina, lo que conduce a un desajuste de ADN y rupturas de doble cadena, que consecuentemente culmina en la muerte de las células afectadas. Cuando la enzima MGMT está activa, evita esta cascada al eliminar el grupo metilo de la guanina O6. La metilación del promotor MGMT da como resultado una disminución de la actividad enzimática de MGMT, lo que conduce a una reparación disminuida del desajuste de ADN y una mayor sensibilidad a TMZ(27).

Múltiples ensayos clínicos en fase III han

\section{(c) $)(1) \Theta$}


evidenciado que la metilación del promotor MGMT confiere una supervivencia más larga de hasta un $50 \%$ en pacientes con GBM tratados con TMZ. Por el contario, en ausencia de metilación del MGMT, existe poca o ninguna respuesta a la quimioterapia con TMZ. Esto podría ser suficiente para la exclusión de esta terapia, no obstante, los estudios aún se encuentran en curso, al igual que otras opciones de terapiagénica dirigida.(10)

La metilación del promotor MGMT es común en los glioblastomas adultos (40\%) y se observa con frecuencia en gliomas IDH mutados / CIMPalto. Dicha metilación del promotor MGMT rara vez se ve en tumores pediátricos. La interacción entre el estado de metilación del promotor MGMT y TMZ es compleja y puede verse afectada por otros factores. Por ejemplo, se han sugerido mutaciones o expresión alterada en las proteínas de reparación de anomalías (MMR) (MSH2, MSH6, MLH1, PMS2) como mecanismos alternativos de resistencia a TMZ. Además, diferentes modalidades de prueba de MGMT, incluyendo IHC, PCR y la pirosecuenciación han arrojado diversos niveles de correlación con el resultado clínico(6).

\section{CONCLUSIÓN}

El GBM es la neoplasia maligna primaria del Sistema Nervioso Central más frecuentemente diagnosticada en Costa Rica y el mundo. Si bien su incidencia es relativamente baja, contribuye de manera desproporcional a la morbimortalidad por cáncer. La expectativa de vida a 5 años, el periodo libre de enfermedad y el estándar de tratamiento continúan sin cambios significativos a pesar de los avances moleculares y en terapia dirigida. Existe gran cantidad de literatura y ensayos clínicos en curso que presentan terapias prometedoras, sin suficiente sustento para su implementación como terapia estándar.
Previo a la integración molecular-histológica para la clasificación de neoplasias del sistema nervioso central, algunos tumores eran clasificados como gliomas de bajo grado a pesar de guardar características moleculares comunes con el GMB. Al determinarse que tumores originarios de glía tienen alteraciones en las vías de señalización comunes para GBM, múltiples estudios sustentan que tendrán un comportamiento similar, así como una misma respuesta al tratamiento.

Es necesario implementar la clasificación establecida por la OMS en 2016 de manera exhaustivaparaoptimizarelabordaje, tratamiento y mejorar el pronóstico de los pacientes. Para tal efecto, podrían implementarse, además de los integrados como criterios, biomarcadores como el MGMT para determinar el estado de metilación del GBM y así predecir la resistencia la Temozolomida, evitando la supradosificación y quimiotoxicidad para los pacientes. Toda atención debe dirigirse a mejorar su calidad de vida, especialmente en el contexto de esta neoplasia de pronóstico exiguo a corto plazo. 


\section{BIBLIOGRAFÍA}

1. Ostrom QT, Cioffi G, Gittleman H, Patil N, Waite K, Kruchko C, et al. CBTRUS Statistical Report: Primary Brain and Other Central Nervous System Tumors Diagnosed in the United States in 2012-2016. Neuro-Oncol. 1 de noviembre de 2019;21(Supplement_5):v1-100.

2. Louis DN, Perry A, Reifenberger G, von Deimling A, Figarella-Branger D, Cavenee WK, et al. The 2016 World Health Organization Classification of Tumors of the Central Nervous System: a summary. Acta Neuropathol (Berl). junio de 2016;131(6):803-20.

3. Zanders ED, Svensson F, Bailey DS. Therapy for glioblastoma: is it working? Drug Discov Today. mayo de 2019;24(5):1193-201.

4. Department of Neurosurgery, Jordan University Hospital and Medical School, University of Jordan, Amman, Jordan, Tamimi AF, Juweid M, Department of Radiology and Nuclear Medicine, Jordan University Hospital and Medical School, University of Jordan, Amman, Jordan. Epidemiology and Outcome of Glioblastoma. En: Department of Neurosurgery, University Hospitals Leuven, Leuven, Belgium, De Vleeschouwer S, editores. Glioblastoma [Internet]. Codon Publications; 2017.

5. Registro Nacional de Tumores. Incidencia de Glioblastomas Según Grupos de Edad por Sexo y Año de Ocurrencia (cifras absolutas), Costa Rica, 2011-2015. Ministerio de Salud de Costa Rica;

6. Brem S, Abdullah KG. Glioblastoma [Internet]. 2017 [citado 1 de diciembre de 2019]. Disponible en: https://www.clinicalkey.com/\#!/browse/book/3-s2.0-C20150059779

7. Schwartz SI, Brunicardi FC, Andersen DK. Principios de cirugía [de] Schwartz. México: McGrawHill Interamericana; 2015.

8. Louis DN, Ohgaki H, Wiestler OD, Cavenee WK, Burger PC, Jouvet A, et al. The 2007 WHO Classification of Tumours of the Central Nervous System. Acta Neuropathol (Berl). 12 de julio de 2007;114(2):97-109.

9. Komori T. The 2016 WHO Classification of Tumours of the Central Nervous System: The Major Points of Revision. Neurol Med Chir (Tokyo). 2017;57(7):301-11.

10. Wen PY, Weller M, Lee EQ, Alexander BA, Barnholtz-Sloan JS, Barthel FP, et al. Glioblastoma in Adults: A Society for Neuro-Oncology (SNO) and European Society of Neuro-Oncology (EANO) Consensus Review on Current Management and Future Directions. Neuro-Oncol. 24 de abril de 2020;noaa106.

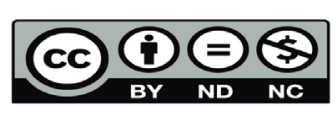


11. Liu Y-Q, Wu F, Li J-J, Li Y-F, Liu X, Wang Z, et al. Gene Expression Profiling Stratifies IDHWildtype Glioblastoma With Distinct Prognoses. Front Oncol. 17 de diciembre de 2019;9:1433.

12. Molenaar RJ, Maciejewski JP, Wilmink JW, van Noorden CJF. Wild-type and mutated IDH1/2 enzymes and therapy responses. Oncogene. abril de 2018;37(15):1949-60.

13. Aldape K, Zadeh G, Mansouri S, Reifenberger G, von Deimling A. Glioblastoma: pathology, molecular mechanisms and markers. Acta Neuropathol (Berl). junio de 2015;129(6):829-48.

14. Malta TM, de Souza CF, Sabedot TS, Silva TC, Mosella MS, Kalkanis SN, et al. Glioma CpG island methylator phenotype (G-CIMP): biological and clinical implications. Neuro-Oncol. 9 de abril de 2018;20(5):608-20.

15. Takeuchi H, Kitai R, Hosoda T, Yamada S, Hashimoto N, Kikuta K, et al. Clinicopathologic features of small cell glioblastomas. J Neurooncol. abril de 2016;127(2):337-44.

16. Brennan CW, Verhaak RGW, McKenna A, Campos B, Noushmehr H, Salama SR, et al. The Somatic Genomic Landscape of Glioblastoma. Cell. octubre de 2013;155(2):462-77.

17. Pekmezci M, Perry A. Practical Molecular Pathologic Diagnosis of Infiltrating Gliomas. Surg Pathol Clin. marzo de 2015;8(1):49-61.

18. Purkait S, Jha P, Sharma MC, Suri V, Sharma M, Kale SS, et al. CDKN2A deletion in pediatric versus adult glioblastomas and predictive value of p16 immunohistochemistry: CDKN2A/p16 in glioblastomas. Neuropathology. agosto de 2013;33(4):405-12.

19. Goldhoff P, Clarke J, Smirnov I, Berger MS, Prados MD, James CD, et al. Clinical Stratification of Glioblastoma Based on Alterations in Retinoblastoma Tumor Suppressor Protein (RB1) and Association With the Proneural Subtype. J Neuropathol Exp Neurol. enero de 2012;71(1):83-9.

20. The Cancer GenomeAtlas Research Network. Comprehensive genomic characterization defines human glioblastoma genes and core pathways. Nature. octubre de 2008;455(7216):1061-8.

21. Trusolino L, Bertotti A, Comoglio PM. MET signalling: principles and functions in development, organ regeneration and cancer. Nat Rev Mol Cell Biol. diciembre de 2010;11(12):834-48.

22. Kong D-S, Song S-Y, Kim D-H, Joo KM, Yoo J-S, Koh JS, et al. Prognostic significance of c-Met expression in glioblastomas: c-Met Expression in Glioblastomas. Cancer. 1 de enero de 2009;115(1):140-8.

23. Ohgaki H. Genetic pathways to glioblastomas. Neuropathology. marzo de 2005;25(1):1-7. 
24. Carico C, Nuño M, Mukherjee D, Elramsisy A, Dantis J, Hu J, et al. Loss of PTEN Is Not Associated with Poor Survival in Newly Diagnosed Glioblastoma Patients of the Temozolomide Era. Lo AWI, editor. PLoS ONE. 29 de marzo de 2012;7(3):e33684.

25. Li X, Wu C, Chen N, Gu H, Yen A, Cao L, et al. PI3K/Akt/mTOR signaling pathway and targeted therapy for glioblastoma. Oncotarget [Internet]. 31 de mayo de 2016 [citado 3 de diciembre de 2019];7(22). Disponible en: http://www.oncotarget.com/fulltext/7961

26. Stupp R, Mason WP, van den Bent MJ, Weller M, Fisher B, Taphoorn MJB, et al. Radiotherapy plus Concomitant and Adjuvant Temozolomide for Glioblastoma. N Engl J Med. 10 de marzo de 2005;352(10):987-96.

27. Jiang $\mathrm{H}$, Zeng $\mathrm{W}$, Ren $\mathrm{X}$, Cui $\mathrm{Y}$, Li M, Yang K, et al. Super-early initiation of temozolomide prolongs the survival of glioblastoma patients without gross-total resection: a retrospective cohort study. J Neurooncol. agosto de 2019;144(1):127-35. 